\title{
Rates of Mortality in Lumbar Spine Surgery and Factors Associated With Its Occurrence Over a 10-Year Period: A Study of 803,949 Patients in the Nationwide Inpatient Sample
}

GREGORY WYATT POORMAN, BA, JOHN Y. MOON, BS, CHARLES WANG, BS, SAMANTHA R. HORN, BA, BRYAN M. BEAUBRUN, BA, OLIVIA J. BONO, BA, ANNE-MARIE FRANCIS, BA, CYRUS M. JALAI, BA, PETER G. PASSIAS, MD

NYU Langone Medical Center Hospital for Joint Diseases New York, New York

\begin{abstract}
Background: The rate of mortality in surgical procedures involving the lumbar spine has historically been low, and as a result, there has been difficulty providing accurate quantitative mortality rates to patients in the preoperative planning phase. Awareness of these mortality rates is essential in reducing postoperative complications and improving outcomes. Additionally, mortality rates can be influenced by procedure type and patient profile, including demographics and comorbidities. The purpose of this study is to assess rates and risk factors associated with mortality in surgical procedures involving the lumbar spine using a large national database.

Methods: The Nationwide Inpatient Sample database was reviewed from 2003 to 2012. A total of 803,949 patients age 18 years or older were identified by ICD-9CM procedure codes for spinal fusion or decompression of the lumbar spine. Mortality was stratified based on type of procedure (simple or complex fusion, decompression), patient demographics and comorbidities, and in-hospital complications. Binary logistic regression was used to identify the risk of death while controlling for comorbidities, race, sex, and procedure performed. Significance was defined as $P<.05$ differences relative to the overall cohort.

Results: Mortality for all patients requiring surgery of the lumbar spine was $0.13 \%$. Mortality based on procedure type was $0.105 \%$ for simple fusions, $0.321 \%$ for complex fusions, and $0.081 \%$ for decompression only. Increased mortality was observed demographically in patients who were male (odds ratio [OR]: 1.75; 95\% confidence interval [CI]: 1.51-2.03), black (OR: 1.40; CI: 1.10-1.79), ages 65-74 (OR: 1.46; CI: 1.25-1.70), and age 75+(OR: 2.70; CI: 2.30-3.17). Comorbidities associated with the greatest increase in mortality were mild (OR: 10.04; CI: 7.76-13.01) and severe (OR: 26.47; CI: 16.03-43.70) liver disease and congestive heart failure (OR: 4.57; CI: 3.77-5.53). The complications with the highest mortality rates were shock (OR: 20.67; CI: 13.89-30.56) and pulmonary embolism (OR: 20.15; CI: 14.01-29.00).

Conclusions: From 2003 to 2012, the overall mortality rate in 803,949 lumbar spine surgery patients was $0.13 \%$. Risk factors that were significantly associated with increased mortality rates were male gender, black race, and ages 6574 and 75+. Comorbidities associated with an increased mortality rate were mild and severe liver disease and congestive heart failure. Inpatient complications with the highest mortality rates were shock and pulmonary embolism. These findings can be helpful to surgeons providing preoperative counseling for patients considering elective lumbar procedures and for allocating resources to treat and prevent perioperative complications leading to mortality.
\end{abstract}

Level of Evidence: 3.

Lumbar Spine

Keywords: lumbar spine surgery, mortality incidence, NIS database

\section{INTRODUCTION}

Orthopaedic procedures have a small but important risk for mortality as a consequence of surgical intervention. Quantification of mortality rates is vital when providing preoperative counseling and weighing risks and benefits with patients. Unfortunately, capturing true mortality rates may be difficult given their uncommon occurrence and lack of sufficiently large sample populations. Further, with the increasing age of the population and number of procedures being performed each year, ${ }^{1}$ more broadly established mortality incidence is needed to identify patient populations at risk for this devastating complication. 
Complications after lumbar spine surgery have received more attention, as postoperative morbidity is trending upward with the number of procedures being performed ${ }^{1}$ and are thus better reviewed. ${ }^{2-4}$ Mortalities after lumbar spine surgeries have been reported in the context of many specific complications: lumbar fusions for elderly patients with renal disease (mortality $1.1 \%$ ), elderly patients after lumbar trauma (mortality $6.8 \%$ ), and adult spinal deformity patients (mortality $0.18 \%) .^{5-7}$ Risk factors most frequently associated with mortality include but are not limited to patient age, sex, gender, and comorbidity status. ${ }^{5,8-11}$ However, literature that evaluates mortality in lumbar spine surgery has traditionally evaluated specific comorbidity populations or spine surgeries. Data from a large database with the ability to consolidate risk factors that should be identified preoperatively in patients are limited or dated. $^{12}$

This study aims to capture nationwide trends in mortality with further stratification based on different procedure types, patient demographics and comorbidities, and in-hospital complications. By examining data on multiple procedures, the current study aims to obtain a broader perspective on mortality in this patient population. Data from a national database will importantly allow for generalizable mortality incidence given the capture of wide geographic regions, hospital types, surgeon characteristics, and patient populations.

\section{METHODS}

\section{Data Source}

The Nationwide Inpatient Sample (NIS) is part of a family of databases developed for the Healthcare Cost and Utilization Project (HCUP) and is the largest publicly available all-payer inpatient health care database in the United States. It provides an approximated $20 \%$ stratified sample of all discharges from US community hospitals and draws from around 8 million hospital stays each year. For each patient, it provides data elements such as demographics; primary or secondary diagnoses and procedures in International Classification of Diseases, Ninth Revision, Clinical Modification (ICD9CM) format; total charges; length of stay; and comorbidity measures. National estimates were generated using discharge weight files. More detailed information is available at https://www.hcup-
us.ahrq.gov/db/nation/nis/NIS_Introduction_2011. pdf. $^{13}$

\section{Inclusion and Exclusion Criteria}

This study is a retrospective review of the NIS database from 2003 to 2012. It includes patients over the age of 18 who were identified by ICD-9CM codes for procedures on the lumbar spine: spinal fusion (81.04-81.08, 81.34-81.38), laminectomy (03.02), discectomy $(80.50,80.51)$, or other decompressions (03.09).

\section{Mortality Predictors}

Demographics that were available on the NIS included gender, race, and age (categorized into groups to facilitate clinical relevance; 18-40, 41-54, 55-64, 65-74, and 75+). Spinal fusion was classified as simple (fewer than 3 levels fused) and complex (3 or more levels or combined approach). Additional procedural variables assessed included decompression-only procedures (excluding patients that received fusion), procedures utilizing any interbody device placement (84.51), and procedures utilizing any bone morphogenetic protein (BMP; 84.52). Concurrent complications (though without any ability to establish a causative relationship with mortality) were queried following previously established protocols analyzing the nationwide inpatient sample. (Full list of ICD-9 coding available in Appendix A.) Comorbidities were available using ICD-9 scoring schema established for the Charlson Comorbidity Index (CCI). Patients' primary diagnosis codes were compared relative to mortality. (Full list of ICD-9 Diagnoses available in Appendix B.)

\section{Statistical Analyses}

Incidence of mortality, calculated using HCUP provided hospital and year adjusted weights, was stratified based on patient demographics, type of procedure, inpatient complications, patient comorbidities, and primary diagnosis. Trend analysis by analysis of variance was performed across the years studied to determine a significant decrease or increase in mortality rate. After establishing incidence, binary logistic regression was used to identify the risk of death while controlling for comorbidities, race, sex, and procedure performed. Significance was defined as $P<.05$ differences relative to the overall cohort. Odds ratios (OR) are reported with 


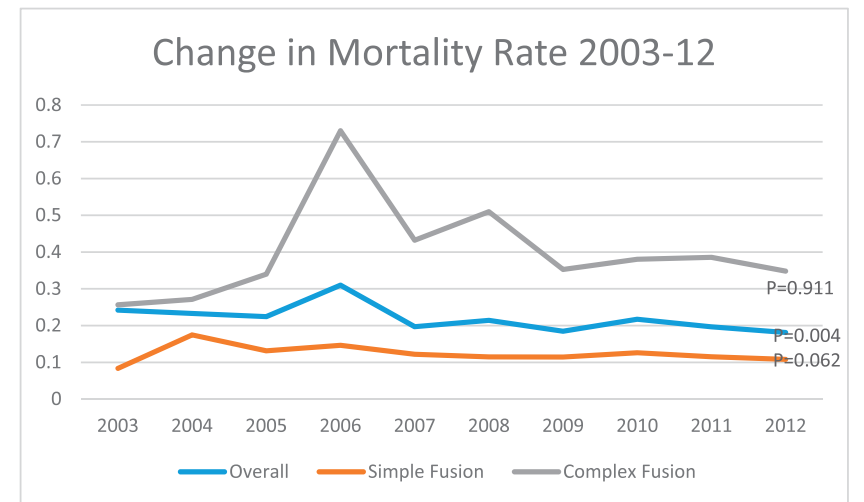

Figure 1. Results of trend analysis by analysis of variance, describing a significant change in mortality rate over 2003-2012.

95\% confidence intervals (CI). IBM SPSS Statistics version 23.0 (IBM Corp, Armonk, New York) was used to perform all descriptive and comparative statistics.

\section{RESULTS}

A total of 803,949 patients $(51.1 \%$ female; average age 53.1 years) undergoing lumbar spine procedures between 2003 and 2012 were identified in the NIS database. There were a total of 1068 mortalities reported. Overall mortality in lumbar spine procedures across the years 2003-2012 was found to be $0.13 \%$. In temporal analysis, there was a significant decrease in the overall mortality rate from 2003 to 2012 (2003: $0.24 \%$; 2012: $0.18 \% ; P=$ .004) (Figure 1).

\section{Demographics}

Male gender was an independent risk factor for mortality (OR: 1.75; CI: 1.51-2.03; Table 1). Ethnically, blacks experienced increased risk of mortality (OR: 1.40; CI: 1.10-1.79]), and whites experienced a protective effect against mortality (OR: 0.79; CI: 0.70-0.91). In analysis of different age-groups, age-groups 18-40, 41-54, and 55-64 had a relatively lower risk for mortality $(\mathrm{OR}<1$, all significant), while ages $65-74$ and $75+$ were at a comparatively increased risk for death $(\mathrm{OR}>1$, all significant).

\section{Procedure}

Mortality rate was $0.10 \%$ in simple fusions, $0.32 \%$ in complex fusions, and $0.08 \%$ in decompression-only procedures (Table 2). Complex posterior-approach fusions (OR: 1.64; CI: 1.38-1.95) and complex combined-approach fusions (OR: 1.47;
Table 1. Rates of mortality stratified by demographics.

\begin{tabular}{lcccc}
\hline Demographic & $\mathbf{N}$ & No. of Deaths & Mortality & Multivariate \\
\hline Overall & 803949 & 1068 & $0.13 \%$ & \\
Gender & & & & \\
$\quad$ Male & 391440 & 620 & $0.16 \%$ & $1.75(1.51-2.03)$ \\
$\quad$ Female & 410629 & 448 & $0.11 \%$ & $0.57(0.49-0.66)$ \\
Race & & & & \\
$\quad$ White & 529044 & 704 & $0.13 \%$ & $0.79(0.70-0.91)$ \\
$\quad$ Black & 39988 & 72 & $0.18 \%$ & $1.40(1.10-1.79)$ \\
Asian & 36177 & 54 & $0.15 \%$ & $1.21(0.90-1.61)$ \\
Other & 199808 & 238 & $0.12 \%$ & $1.14(0.98-1.32)$ \\
Age & & & & \\
18-40 & 137727 & 33 & $0.02 \%$ & $0.24(0.16-0.37)$ \\
$41-54$ & 221353 & 118 & $0.05 \%$ & $0.48(0.39-0.59)$ \\
$55-64$ & 172157 & 195 & $0.11 \%$ & $0.82(0.96-0.98)$ \\
65-74 & 166466 & 341 & $0.20 \%$ & $1.46(1.25-1.70)$ \\
$75+$ & 107306 & 380 & $0.35 \%$ & $2.70(2.30-3.17)$ \\
& & & & \\
\hline
\end{tabular}

CI: 1.20-1.81) demonstrated the highest increase of risk. Neither interbody device procedures (OR: 0.69; CI: 0.60-0.80]) nor BMP procedures (OR: 0.70; CI: 0.59-0.83) increased risk of mortality. Simple fusions displayed a significantly lower risk for death (OR: 0.39; CI: 0.33-0.45).

\section{Concurrent Complications}

Shock (OR: 20.67; CI: 13.89-30.56) and pulmonary embolism (OR: 20.15; CI: 14.01-29.00) recorded the highest increase in risk of death-over 20 times for each (Table 3). acute respiratory distress syndrome (OR: 11.91; CI: 9.89-14.35), cardiacrelated complications (OR: 8.69; CI: 6.90-10.96), and deep-vein thrombosis (OR: 7.42; CI: 5.64-9.76) followed. Other in-hospital complications that were associated with increased risk of mortality included nervous system complications, peripheral vascular disease, respiratory system complications, digestive system complications, and infections (OR $>1$, all significant).

\section{Patient Comorbidities}

The comorbidities associated with the highest mortality risk were mild (OR: 10.04; CI: 7.76-13.01) or moderate/severe (OR: 26.47; CI: 16.03-43.70) liver diseases. Metastatic solid tumor diagnosis (OR: 6.28; CI: 5.08-7.76) increased mortality risk significantly. However, malignancy (OR: 1.13; CI: 0.861.49) did not increase risk of death after controlling for patients with both malignancy and metastatic tumor status. Following liver disease and metastatic tumor, congestive heart failure (OR: 4.57; CI: 3.775.53), cerebrovascular disease (OR: 3.92; CI: 2.965.19), and previous myocardial infarction (OR: 3.50 ; CI: 2.87-4.26) were the riskiest comorbidities in 
Table 2. Rates of mortality stratified by type of procedure.

\begin{tabular}{|c|c|c|c|c|}
\hline Procedure & $\mathbf{N}$ & No. of Deaths & Mortality & Multivariate \\
\hline Simple fusion & 304970 & 320 & $0.10 \%$ & $0.39(0.33-0.45)$ \\
\hline Anterior & 103263 & 121 & $0.12 \%$ & $0.62(0.51-0.76)$ \\
\hline Posterior & 198727 & 198 & $0.10 \%$ & $0.48(0.41-0.57)$ \\
\hline Complex fusion ( $3+$ levels or combined approach) & 104784 & 336 & $0.32 \%$ & $1.77(1.53-2.05)$ \\
\hline Anterior & 13515 & 46 & $0.34 \%$ & $1.23(0.91-1.67)$ \\
\hline Posterior & 44619 & 180 & $0.40 \%$ & $1.64(1.38-1.95)$ \\
\hline Combined & 45924 & 106 & $0.23 \%$ & $1.47(1.20-1.81)$ \\
\hline Decompression only & 364494 & 297 & $0.08 \%$ & $1.31(1.14-1.50)$ \\
\hline Interbody device & 244327 & 286 & $0.12 \%$ & $0.69(0.60-0.80)$ \\
\hline Bone morphogenetic protein & 148022 & 180 & $0.12 \%$ & $0.70(0.59-0.83)$ \\
\hline
\end{tabular}

mortality analysis. Dementia, rheumatic disease, and diabetes did not carry any significant additional risk of death.

\section{DISCUSSION}

While mortality incidence in lumbar surgery is low, a fundamental aspect of preoperative counseling is educating the patient on risks associated with surgery based on evidence-based literature. For reference, the Centers for Disease Control and Prevention (CDC) reported a $0.81 \%$ overall mortality rate in 2011 for the general population of the United States. ${ }^{14}$ Patient comprehension of riskbenefit discussions can be enhanced with the use of quantitative values over statements, but despite the importance of mortality risk education, these values can be difficult to obtain for rare events such as death. Literature on informed consent found that patients not counseled using a numeric approach had a tendency to overestimate benefits and underestimate risk. ${ }^{15,16}$ Therefore, the mortality incidence established in lumbar surgeries stratified by patient comorbidities and procedures undergone may be particularly useful for patient counsel.
Mortality incidences established by the current studies' data describe expected risk factors: increasing age, male gender, black race, more complex surgeries performed, concurrent complications, and certain comorbidities. These risk factors were isolated as independent risk factors by controlling for comorbidities, race, sex, and procedure performed (except when testing the respective factors). Increasing age as an independent risk factor is consistent with findings of an important earlier study by Deyo et $\mathrm{al}^{12}$ that also looked at mortality in lumbar surgery but was limited to a single institution. A study by Schoenfeld et $\mathrm{al}^{8}$ used pooled data to examine the influence of patient sex on morbidity and mortality in spine surgery and found that males were at increased risk compared to females. This was not well characterized in lumbar spine procedures but our analysis suggests that this is applicable specifically to the lumbar spine as well. Further research is necessary to identify the etiologies responsible for the differences in mortality risk that are being observed by demographics. Black race is an established risk factor across many surgical specialties including spine. ${ }^{9}$ The reasons for this are multifactorial and may include health

Table 3. Rates of mortality stratified by complication.

\begin{tabular}{|c|c|c|c|c|}
\hline Complication & $\mathbf{N}$ & No. of Deaths & Mortality & Odds Ratio \\
\hline Dysphagea & 605 & 8 & $1.32 \%$ & $2.05(0.72-2.83)$ \\
\hline Nervous system & 6564 & 72 & $1.10 \%$ & $4.29(3.34-6.07)$ \\
\hline Cardiac complication & 5110 & 153 & $2.99 \%$ & $8.69(6.90-10.96)$ \\
\hline Peripheral vascular disease & 488 & 9 & $1.84 \%$ & $3.13(1.14-8.59)$ \\
\hline Respiratory & 5683 & 40 & $0.70 \%$ & $2.58(1.76-3.80)$ \\
\hline Digestive & 9485 & 44 & $0.46 \%$ & $1.66(1.12-2.45)$ \\
\hline Urinary & 7416 & 19 & $0.26 \%$ & $1.45(0.85-$ \\
\hline Device & 25104 & 29 & $0.12 \%$ & $0.65(0.44-0.96)$ \\
\hline Shock & 432 & 39 & $9.03 \%$ & $20.67(13.89-30.56)$ \\
\hline Hematoma & 5202 & 36 & $0.69 \%$ & $2.00(1.24-3.22)$ \\
\hline Puncture & 17773 & 56 & $0.32 \%$ & $1.18(0.78-1.80)$ \\
\hline Infection & 3509 & 53 & $1.51 \%$ & $6.92(4.99-9.59)$ \\
\hline Anemia & 61644 & 182 & $0.30 \%$ & $0.60(0.50-0.73)$ \\
\hline Acute respiratory distress syndrome & 5618 & 226 & $4.02 \%$ & $11.91(9.89-14.35)$ \\
\hline Pulmonary embolism & 959 & 53 & $5.53 \%$ & $20.15(14.01-29.00)$ \\
\hline Deep-vein thrombosis & 3058 & 94 & $3.07 \%$ & $7.42(5.64-9.76)$ \\
\hline
\end{tabular}


Table 4. Rates of mortality stratified by comorbidities.

\begin{tabular}{|c|c|c|c|c|}
\hline Comorbidity & $\mathbf{N}$ & No. of Deaths & Mortality (\%) & $\mathbf{P}$ \\
\hline Previous myocardial infarction & 26231 & 193 & $0.74 \%$ & $3.50(2.87-4.26)$ \\
\hline Congestive heart failure & 16683 & 237 & $1.42 \%$ & $4.57(3.77-5.53)$ \\
\hline Peripheral vascular disease & 15968 & 87 & $0.54 \%$ & $1.47(1.06-2.04)$ \\
\hline Cerebrovascular disease & 9238 & 101 & $1.09 \%$ & $3.92(2.96-5.19)$ \\
\hline Dementia & 1187 & 7 & $0.59 \%$ & $1.32(0.44-3.46)$ \\
\hline Chronic pulmonary disease & 107005 & 206 & $0.19 \%$ & $1.24(1.04-1.49)$ \\
\hline Rheumatic disease & 20080 & 23 & $0.11 \%$ & $0.55(0.31-0.95)$ \\
\hline Peptic ulcer disease & 3978 & 17 & $0.43 \%$ & $2.96(1.80-4.86)$ \\
\hline Mild liver disease & 5183 & 99 & $1.91 \%$ & $10.04(7.76-13.01)$ \\
\hline Diabetes without chronic complication & 115559 & 150 & $0.13 \%$ & $0.60(0.48-0.75)$ \\
\hline Diabetes with chronic complication & 10102 & 40 & $0.40 \%$ & $1.40(0.91-2.13)$ \\
\hline Hemiplegia or paraplegia & 15993 & 102 & $0.64 \%$ & $2.32(1.80-3.00)$ \\
\hline Renal disease & 72140 & 276 & $0.38 \%$ & $1.44(1.22-1.70)$ \\
\hline Moderate or severe liver disease & 342 & 27 & $7.89 \%$ & $26.47(16.03-43.70)$ \\
\hline Any malignancy & 11796 & 228 & $1.93 \%$ & $1.13(0.86-1.49)$ \\
\hline Metastatic solid tumor & 9504 & 336 & $3.54 \%$ & $6.28(5.08-7.76)$ \\
\hline
\end{tabular}

care access and utilization in addition to anatomic differences. Skolasky et $\mathrm{al}^{9}$ reported an OR of 1.59 (CI 1.30-2.96) in analyzing additional risk of mortality in cervical spine surgery in blacks relative to whites, comparable to our finding of 1.40 (CI 1.10-1.79).

The results obtained on patient comorbidities suggest that liver disease is associated with a significantly increased mortality rate. This may be related to the liver's multiple physiologic roles, including coagulation and its effect on blood loss. A large study looking at perioperative morbidity and mortality in spinal fusion found that the highest risk of mortality was associated with a finding of coagulopathy. ${ }^{17}$ This may be compatible with our finding of liver disease and increased mortality. Congestive heart failure and history of myocardial infarction as predictors for mortality in noncardiac surgery is unsurprising, as they are well established. ${ }^{18-20}$ A history of cerebrovascular disease was also identified as a significant patient comorbidity associated with mortality. This is consistent with the findings of Jorgensen et $\mathrm{al}^{21,22}$ in a novel study that found a history of stroke to be associated with adverse outcomes after surgery. Their recommendation to wait 9 months in this patient population until elective surgery may be warranted. Interestingly, controlling for metastatic tumor diagnosis caused malignancy diagnoses to remain insignificant as a mortality risk factor. Malignancies included lymphoma and leukemia but excluded malignant neoplasms of the skin.

A few comorbidities stood out with large patient cohorts and high mortality: 5183 patients with mild liver disease sustained mortality of $1.91 \%, 16,683$ congestive heart failure patients sustained mortality of $1.42 \%$, and 9238 patients with cerebrovascular disease sustained mortality of $1.09 \%$. These patient groups, with high risk of death, deserve further study to establish if the risk factors established by the current study, ranging from 3.92 to 10.04 , could have been avoided via different or nonsurgical treatment.

In analyzing diagnostic risk factors for mortality in lumbar spine surgery, disc displacement, stenosis, disc degeneration, and spondylolisthesis, by far the highest-volume surgeries, demonstrated decreased risk relative to surgeries such as fracture, scoliosis, and cord injuries. After controlling for the type of surgery being performed (simple fusion, complex fusion, or just decompression), these high-volume surgeries continued to show decreased risk of death

Table 5. Rates of mortality stratified by diagnosis.

\begin{tabular}{lrrr}
\hline Diagnosis & N & No. of Deaths & Mortality \\
\hline Disc displacement & 169366 & 158 & $0.09 \%$ \\
Stenosis & 176653 & 246 & $0.14 \%$ \\
Disc degeneration & 225245 & 194 & $0.09 \%$ \\
Spondylolisthesis & 117872 & 123 & $0.62(0.49-0.78)$ \\
Postlaminectomy syndrome & 24544 & 26 & $0.48(0.40-0.58)$ \\
Fracture & 24560 & 245 & $0.47(0.38-0.58)$ \\
Scoliosis & 64548 & 176 & $0.99 \%$ \\
Sacroilitis & 926 & 1 & $0.27 \%$ \\
Cord injury & 235 & 6 & $0.53(0.39-0.64)$ \\
& & $0.83)$ & $0.11 \%$ \\
\hline
\end{tabular}


relative to less frequent, commonly more complex surgeries. Surprisingly, surgeries associated with a scoliosis diagnosis were not associated with an increased risk of death. This defies expectations, as scoliosis is frequently associated with deformity correction and a plethora of comorbid factors but is backed up by literature. For example, Smith et $\mathrm{al}^{23}$ reported mortality incidence of only 1.8 per 1000 in over 100000 deformity surgeries.

The limitations to our study arise from the design of the NIS database. Procedure type was divided to roughly stratify case complexity, but the available data prevent us from detailing the surgical procedure (ie, estimated blood loss, surgery length, preoperative American Society of Anaesthesiologists grade) and identifying confounders for mortality. In addition, patient comorbidities are not fully specified (eg, severity, length of disease, patient medication compliance). Another limitation to recognize is that the severity of complications is not quantified by the NIS, with the end point noted only if it involved mortality. Also, due to our data analysis procedure, the operative diagnosis was not examined for many groups. The operative diagnosis could explain, for example, the justification for operation on patients with liver disease or metastatic cancer. Finally, because the NIS database is an inpatient sample, it is unable to capture complications and death in the postoperative period following discharge. While this underestimates the overall mortality rate, the Scoliosis Research Society database, including approximately 100000 cases, found that close to $80 \%$ of postoperative mortality occurred during the patient's initial hospital stay. ${ }^{23}$ This study is applicable to elective procedures but cannot be generalized to all lumbar surgeries because it does not include emergency admissions or cases involving trauma, fracture, or malignancy. This underestimates the overall mortality associated with lumbar spine surgery, and our findings should be interpreted in the provided context. An earlier study of the NIS from 1998 to 2008 by Memtsoudis et $\mathrm{al}^{11}$ included both emergent and urgent cases and found a higher mortality rate of $0.20 \%$ in patients undergoing lumbar spinal fusion.

\section{CONCLUSIONS}

Between 2003 and 2012, there was an overall mortality incidence rate of $0.13 \%$. After controlling for comorbidities, race, sex, and procedure performed, increased risk of mortality was seen with complex fusion and older age, male sex, and/or black race. Significant comorbidities associated with increased risk included a history of liver disease, congestive heart failure, and cerebrovascular disease. These results may be helpful to surgeons in determining risk prognosis and identifying high-risk patients prior to lumbar surgical procedures with the hopes of improving outcomes and reducing patient mortality.

\section{REFERENCES}

1. Goz V, Weinreb JH, McCarthy I, Schwab F, Lafage V, Errico TJ. Perioperative complications and mortality after spinal fusions. Spine (Phila Pa 1976). 2013;38(22):1970-1976. https://doi.org/10.1097/BRS.0b013e3182a62527.

2. Fineberg SJ, Ahmadinia K, Patel AA, Oglesby M, Singh $\mathrm{K}$. Incidence and mortality of cardiac events in lumbar spine surgery. Spine (Phila Pa 1976). 2013;38(16):1422-1429. https:// doi.org/10.1097/BRS.0b013e3182986d71.

3. Fineberg SJ, Oglesby M, Patel AA, Pelton M a, Singh K. The incidence and mortality of thromboembolic events in lumbar spine surgery. Spine (Phila Pa 1976). 2013;38(13):1154 1159. https://doi.org/10.1097/BRS.0b013e318286b7c0.

4. Marquez-Lara A, Nandyala SV, Fineberg SJ, Singh K. Cerebral vascular accidents after lumbar spine fusion. Spine (Phila Pa 1976). 2014;39(8):673-677. https://doi.org/10.1097/ BRS.0000000000000197.

5. Smith JS, Saulle D, Chen C-J, et al. Rates and causes of mortality associated with spine surgery based on 108,419 procedures: a review of the Scoliosis Research Society Morbidity and Mortality Database. Spine (Phila Pa 1976). 2012;37(23):19751982. https://doi.org/10.1097/BRS.0b013e318257fada.

6. Puvanesarajah V, Jain A, Hess DE, Shimer AL, Shen FH, Hassanzadeh H. Complications and mortality after lumbar spinal fusion in elderly patients with late stage renal disease. Spine (Phila Pa 1976). 2016;41(21):E1298-E1302. https://doi. org/10.1097/BRS.0000000000001618.

7. Winkler EA, Yue JK, Birk $\mathrm{H}$, et al. Perioperative morbidity and mortality after lumbar trauma in the elderly. Neurosurg Focus. 2015;39(4):E2. https://doi.org/10.3171/2015. 7.FOCUS15270.

8. Schoenfeld AJ, Reamer EN, Wynkoop EI, Choi H, Bono CM. Does patient sex affect the rate of mortality and complications after spine surgery? A systematic review. Clin Orthop Relat Res. 2014;473(8):2479-2486. https://doi.org/10. 1007/s11999-014-4102-z.

9. Skolasky RL, Thorpe RJ, Wegener ST, Riley LH. Complications and mortality in cervical spine surgery: racial differences. Spine (Phila Pa 1976). 2014;39(18):1506-1512. https://doi.org/10.1097/BRS.0000000000000429.

10. Cloyd JM, Acosta FL, Cloyd C, Ames CP. Effects of age on perioperative complications of extensive multilevel thoracolumbar spinal fusion surgery. J Neurosurg Spine. 2010;12(4):402408. https://doi.org/10.3171/2009.10.SPINE08741.

11. Memtsoudis SG, Vougioukas VI, Ma Y, Gaber-Baylis LK, Girardi FP. Perioperative morbidity and mortality after anterior, posterior, and anterior/posterior spine fusion surgery. 
Spine (Phila Pa 1976). 2011;36(22):1867-1877. https://doi.org/ 10.1097/BRS.0b013e3181c7decc.

12. Deyo RA, Cherkin DC, Loeser JD, Bigos SJ, Ciol MA. Morbidity and mortality in association with operations on the lumbar spine. The influence of age, diagnosis, and procedure. $J$ Bone Joint Surg Am. 1992;74(4):536-543.

13. Agency for Healthcare Research and Quality. 2011 Introduction to the HCUP Nationwide Inpatient Sample (NIS) 2015;4287.

14. Centers for Disease Control and Prevention, National Center for Health Statistics. Underlying cause of death 19992015. CDC WONDER Online Database. n.d. http://wonder. cdc.gov/ucd-icd10.html. Accessed May 23, 2017.

15. Kalet A, Roberts JC, Fletcher R. How do physicians talk with their patients about risks? J Gen Intern Med. 1994;9(7):402-404. https://doi.org/10.1007/BF02629523.

16. Siminoff LA, Fetting JH, Abeloff MD. Doctor-patient communication about breast cancer adjuvant therapy. J Clin Oncol. 1989;7(9):1192-1200.

17. Shen Y, Silverstein JC, Roth S. In-hospital complications and mortality after elective spinal fusion surgery in the United States: a study of the nationwide inpatient sample from 2001 to 2005. J Neurosurg Anesthesiol. 2009;21(1):21-30. https://doi. org/10.1097/ANA.0b013e31818b47e9.

18. Turrentine FE, Sohn M-W, Jones RS. Congestive heart failure and noncardiac operations: risk of serious morbidity, readmission, reoperation, and mortality. $\mathrm{J} \mathrm{Am}$ Coll Surg. 2016;222(6):1220-1229. https://doi.org/10.1016/j. jamcollsurg.2016.02.025.

19. Hernandez AF, Whellan DJ, Stroud S, Sun JL, O'Connor $\mathrm{CM}$, Jollis JG. Outcomes in heart failure patients after major noncardiac surgery. J Am Coll Cardiol. 2004;44(7):1446-1453. https://doi.org/10.1016/j.jacc.2004.06.059.

20. Goldman L, Caldera DL, Nussbaum SR, et al. Multifactorial index of cardiac risk in noncardiac surgical procedures. N Engl J Med. 1977;297(16):845-850. https://doi. org/10.1097/00132586-197810000-00051.

21. Jørgensen ME, Torp-Pedersen C, Gislason GH, et al. Time elapsed after ischemic stroke and risk of adverse cardiovascular events and mortality following elective noncar- diac surgery. JAMA. 2014;312(3):269-277. https://doi.org/10. 1001/jama.2014.8165.

22. Minhas SV, Goyal P, Patel AA. What are the risk factors for cerebrovascular accidents after elective orthopaedic surgery? Clin Orthop Relat Res. 2016;474(3):611-618. https://doi.org/10. 1007/s11999-015-4496-2.

23. Smith JS, Saulle D, Chen C-J, et al. Rates and causes of mortality associated with spine surgery based on 108,419 procedures: a review of the Scoliosis Research Society Morbidity and Mortality Database. Spine (Phila Pa 1976). 2012;37(23):19751982. https://doi.org/10.1097/BRS.0b013e318257fada.

Disclosures and COI: Gregory Wyatt Poorman, John Y. Moon, Charles Wang, Samantha R. Horn, Bryan M. Beaubrun, Olivia J. Bono, AnneMarie Francis, and Cyrus M. Jalai all report no conflict of interest. Peter G. Passias reports consulting with Medicrea and Zimmer, unrelated to and outside of the current work. All other authors report no conflicts of interest. Given the deidentified nature of the data reported from this study, it is exempt from IRB approval.

Corresponding Author: Peter G. Passias, MD, New York Spine Institute, NYU Medical Center, Hospital for Joint Diseases, Department of Orthopaedic Surgery, 301 East 17th St, New York, NY 10003. Phone: (516) 357-8777; Fax: (516) 3570087; Email: Peter.Passias@nyumc.org.

Published 15 October 2018

This manuscript is generously published free of charge by ISASS, the International Society for the Advancement of Spine Surgery. Copyright (c) 2018 ISASS. To see more or order reprints or permissions, see http://ijssurgery.com. 